\title{
ON POLYGONAL MEASURES WITH VANISHING HARMONIC MOMENTS
}

\author{
DMITRII V. PASECHNIK AND BORIS SHAPIRO
}

To the memory of Andrei Zelevinsky

\begin{abstract}
A polygonal measure is the sum of finitely many real constant density measures supported on triangles in $\mathbb{C}$. Given a finite set $S \subset \mathbb{C}$, we study the existence of polygonal measures spanned by triangles with vertices in $S$, which have all harmonic moments vanishing. For $S$ generic, we show that the dimension of the linear space of such measures is $\left(\begin{array}{c}|S|-3 \\ 2\end{array}\right)$.

We also investigate the situation where the resulting density attains only values 0 or \pm 1 , which corresponds to pairs of polygons of unit density having the same logarithmic potential at $\infty$. We show that such a signed measure does not exist if $|S| \leq 5$, but for each $n \geq 6$ there exists an $S$, with $|S|=n$, giving rise to such a signed measure.
\end{abstract}

\section{INTRODUCTION AND MAIN RESULTS}

Inverse problems in logarithmic potential theory have attracted substantial attention since the publication of the fundamental paper [15, where P.S. Novikov, in particular, proved that two convex (or, more generally, star-shaped) domains in $\mathbb{C}$ with unit density cannot have the same logarithmic potential near $\infty$. Notice that the knowledge of the germ of a logarithmic potential of a finite compactly supported Borel measure $\mu$ at $\infty$ is equivalent to the knowledge of the sequence of its harmonic moments $m_{j}(\mu), j=0,1, \ldots$, where the $j$-th harmonic moment of $\mu$ is defined by:

More precisely, if

$$
m_{j}(\mu)=\int_{\mathbb{C}} z^{j} d \mu(z) .
$$

is the logarithmic potential of $\mu$ and

$$
\mathfrak{u}_{\mu}(z):=\int_{\mathbb{C}} \ln |z-\xi| d \mu(\xi)
$$

$$
\mathfrak{C}_{\mu}(z):=\int_{\mathbb{C}} \frac{d \mu(\xi)}{z-\xi}=\frac{\partial \mathfrak{u}_{\mu}(z)}{\partial z}
$$

is its Cauchy transform then the Taylor expansion of $\mathfrak{C}_{\mu}(z)$ at $\infty$ has the form:

$$
\mathfrak{C}_{\mu}(z)=\frac{m_{0}(\mu)}{z}+\frac{m_{1}(\mu)}{z^{2}}+\frac{m_{2}(\mu)}{z^{3}}+\ldots
$$

Thus Novikov's result can be reformulated as the statement that two convex domains in $\mathbb{C}$ with unit density cannot have coinciding sequences of harmonic moments. It is well-known that already for non-convex domains with unit density the uniqueness in this problem no longer holds. For instance, examples of pairs of non-convex polygons with the same logarithmic potential near $\infty$ can be found on [6. p. 333], see Fig. 1 below. The class of general polygons as well as domains

Date: September 15, 2018.

2010 Mathematics Subject Classification. Primary 44A60; Secondary 31B20.

Key words and phrases. potential theory, harmonic moments, polygonal measures. 
bounded by lemniscates has attracted a substantial attention in this area. Several authors have also considered the class of polynomial densities instead of the unit density.

By a convex polygon we mean the convex hull of finite many points in the plane, at least 3 of which are non-collinear. A general polygon is the set-theoretic union of finitely many convex polygons. By a vertex of a polygon we mean a point of its boundary such that its sufficiently small $\epsilon$-neighborhood in the polygon is different from a half-disk of radius $\epsilon$.

Given an open set $D \subset \mathbb{C}$, define its standard measure

$$
\mu_{D}=\chi_{D} d x d y,
$$

where $\chi_{D}$ is the characteristic function of $D$. The same measure is associated with the closure of $D$. We say that two polygons in $\mathbb{C}$ are equipotential if their standard measures create coinciding logarithmic potential outside their union. Below we present one of the simplest examples of pairs of equipotential polygons given in 6 , Example 1].

Example 1. Consider the 6-tuples $T=\{ \pm \sqrt{3} \pm I, \pm 2 I\}$ and $T^{\prime}=\left\{ \pm \frac{1 \pm \sqrt{3} I}{2}, \pm 1\right\}$. Let $F \subset \mathbb{C}$ be the difference of the convex hull of $T$ and the union of the set of 6 triangles obtained as the orbit of the triangle with nodes $(\sqrt{3}+I, \sqrt{3}-I, 1)$ under the rotation by $\frac{\pi}{3}$, see Fig. 1. Let $F^{\prime} \subset \mathbb{C}$ be the difference of the convex hulls of $T$ and of $T^{\prime}$. Then $F$ and $F^{\prime}$ have the same logarithmic potential.
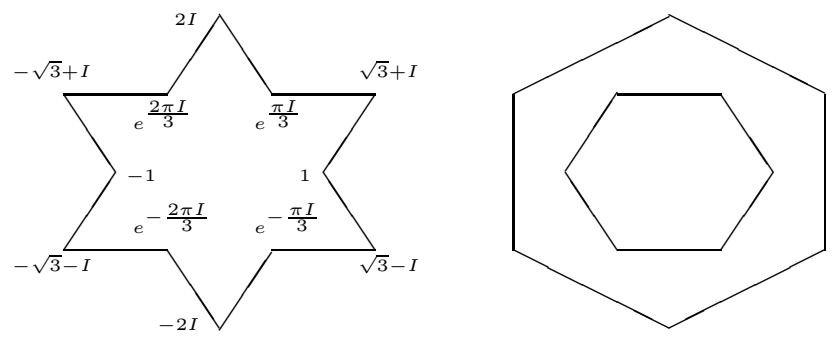

Figure 1. Two equipotential polygons: $F$ on the left, $F^{\prime}$ on the right.

Notice that if different polygons with constant (but not necessarily unit) density have the same logarithmic potential near $\infty$ then they must have the same set of vertices, see [6, Corollary 2 and Lemma 2]. (The coincidence of the logarithmic potential near $\infty$ implies even more restrictions on the polygons than just the coincidence of their set of vertices, cf. [6].)

Taking this fact into account we pose the following classical inverse logarithmic potential problem for polygons in $\mathbb{C}$.

Problem 1. Given a finite set $S \subset \mathbb{C}$, determine whether there exist two equipotential polygons whose sets of vertices coincide with $S$.

One can show that for generic $S$ no pairs of equipotential polygons exist.

Definition 1. A complex (respectively, real) polygonal measure $\mu:=\mu(\mathcal{D})$ is the sum

$$
\mu:=\sum_{\Delta \in \mathcal{D}} c_{\Delta} \mu_{\Delta}, \quad c_{\Delta} \in \mathbb{C}\left(\text { respectively, } c_{\Delta} \in \mathbb{R}\right)
$$

where $\mathcal{D}$ is a finite set of closed triangles in the plane. The set of vertices of the triangles $\Delta \in \mathcal{D}$ with $c_{\Delta} \neq 0$ in (1.1) is called the set of nodes of this decomposition. 
Notice that the decomposition (1.1) of a given $\mu$ need not be unique, and different choices of $\mathcal{D}$ can lead to different sets of nodes.

Besides the nodes of decompositions (1.1) of $\mu$ it is natural to talk about the vertices of $\mu$. They are $v \in \mathbb{C}$ such that for any sufficiently small $\epsilon>0$ the restriction of the density of $\mu$ to the $\epsilon$-disk centered at $v$ is neither constant, nor there exists a line through $v$ dividing the disk into two halves with different constant densities.

Obviously, the set of vertices of $\mu$ is a subset of the set of intersections of sides of the triangles in $\mathcal{D}$. There exists a finite collection $\tilde{\mathcal{D}}$ of triangles with pairwise empty intersections of interiors, such that $\mu=\mu(\tilde{\mathcal{D}})$, and nodes and vertices of $\mu$ coincide. However, such a representation of $\mu$ need not be the most economic one, cf. e.g. Example 1 .

Namely, in notation of Example 1, consider $\tilde{\mu}:=\mu_{F}-\mu_{F^{\prime}}$. Observe that $\tilde{\mu}$ can be represented using only 6 nodes, although the polygons themselves have 12 vertices! This also illustrates the non-uniqueness of representation of $\tilde{\mu}$ in the form (1.1). Indeed,

$$
2 \tilde{\mu}=\sum_{0 \leq j \leq 5} \mu_{\exp \left(\frac{j \pi I}{3}\right)(\sqrt{3}+I, \sqrt{3}-I,-2 I)}-\sum_{0 \leq j \leq 1} \mu_{\left(\sqrt{3}+(-1)^{j} I, \sqrt{3}-(-1)^{j} I,-\sqrt{3}+(-1)^{j} I\right)} .
$$

Let an $S$ admit a pair of equipotential polygons. Taking the difference of their standard measures, one obtains a polygonal measure supported on the convex hull $\operatorname{conv}(S)$ of $S$ with density attaining only values $0, \pm 1$ and with all harmonic moments vanishing. Conversely, if one can find a polygonal measure with all vanishing harmonic moments, and such that its density attains only values $0, \pm 1$, then one obtains a pair of equipotential polygons by taking the differences of $\operatorname{conv}(S)$ with the sets where the density attains value 1 , respectively -1 .

If we weaken the condition that the density of a polygonal measure attains only values $0, \pm 1$ then we arrive at the setup of the present paper. Given a spanning set $S$ (i.e. $S$ contains at least 3 non-collinear points), we introduce the linear spaces $\mathfrak{M}^{\mathbb{R}}(S) \subset \mathfrak{M}^{\mathbb{C}}(S)$ of real-valued, respectively, complex-valued polygonal measures obtained as real, respectively, complex linear spans of the standard measures of all triangles with vertices in $S$. Obviously, $\mathfrak{M}^{\mathbb{C}}(S)=\mathbb{C} \otimes \mathfrak{M}^{\mathbb{R}}(S)$.

We make a further step in the study of (non-)uniqueness in logarithmic potential theory by considering the following question.

Problem 2. Given a finite set $S \subset \mathbb{C}$, determine the linear subspace $\mathfrak{M}_{\text {null }}^{\mathbb{R}}(S) \subset$ $\mathfrak{M}^{\mathbb{R}}(S)$ of real-valued polygonal measures (resp. of complex-valued polygonal measures $\left.\mathfrak{M}_{\text {null }}^{\mathbb{C}}(S) \subset \mathfrak{M}^{\mathbb{C}}(S)\right)$ with all harmonic moments vanishing.

The main technical tool we use is the normalized generating function $\Psi_{\mu}(u)$ for harmonic moments of a measure $\mu$, defined by

$$
\Psi_{\mu}(u)=\sum_{j=0}^{\infty}\left(\begin{array}{c}
j+2 \\
2
\end{array}\right) m_{j}(\mu) u^{j} .
$$

Notice that $\Psi_{\mu}(u)$ is closely related to the Cauchy transform $\mathfrak{C}_{\mu}(z)$ at $\infty$. Namely,

$$
\Psi_{\mu}(u)=\frac{1}{2} \frac{d^{2}}{d u^{2}}\left(\sum_{j=0}^{\infty} m_{j}(\mu) u^{j+2}\right) .
$$

At the same time for a compactly supported measure $\mu$ and sufficiently large $|z|$, $z \mathfrak{C}_{\mu}(z)=\sum_{j=0}^{\infty} m_{j}(\mu) / z^{j}$. Thus for $|u|$ sufficiently small,

$$
\Psi_{\mu}(u)=\frac{1}{2} \frac{d^{2}}{d u^{2}}\left(u \mathfrak{C}_{\mu}\left(\frac{1}{u}\right)\right) .
$$


Similar multivariate generating functions were recently considered in [14. Important in our consideration are the following observations.

Proposition 1. For measures $\mu$ with compact support,

$$
\Psi_{\mu}(u)=\sum_{j=0}^{\infty}\left(\begin{array}{c}
j+2 \\
2
\end{array}\right) m_{j}(\mu) u^{j}=\int \frac{d \mu(z)}{(1-u z)^{3}} .
$$

The normalized generating function $\Psi_{\Delta}(u)$ of (the standard measure of ) the triangle $\Delta \subset \mathbb{C}$ whose vertices are located at $a, b, c$ is given by

$$
\Psi_{\Delta}(u)=\frac{\text { Area } \Delta}{(1-a u)(1-b u)(1-c u)} .
$$

Note that the integral transform in (1.3) appears to be a variant of Fantappiè transformation, cf. [4.

Definition 2. We say that a finite set $S=\left\{z_{0}, z_{1}, \ldots, z_{n}\right\}$ of points in $\mathbb{C}$ is nondegenerate if no three of its points are collinear.

Proposition 2. For any non-degenerate set $S=\left\{z_{0}, z_{1}, \ldots, z_{n}\right\}, n \geq 2$ of points in $\mathbb{C}$ and any fixed non-negative integer $j \leq n$, the set of (standard measures of) all triangles with a node at $z_{j}$ is a basis of the spaces $\mathfrak{M}^{\mathbb{R}}(S)$ and $\mathfrak{M}^{\mathbb{C}}(S)$. In particular,

$$
\operatorname{dim}_{\mathbb{R}} \mathfrak{M}^{\mathbb{R}}(S)=\operatorname{dim}_{\mathbb{C}} \mathfrak{M}^{\mathbb{C}}(S)=\left(\begin{array}{l}
n \\
2
\end{array}\right) .
$$

We are interested in linear subspaces $\mathfrak{M}_{\text {null }}^{\mathbb{R}}(S) \subset \mathfrak{M}^{\mathbb{R}}(S)\left(\right.$ resp. $\mathfrak{M}_{\text {null }}^{\mathbb{C}}(S) \subset$ $\mathfrak{M}^{\mathbb{C}}(S)$ ) of real-valued (resp. complex-valued) measures having all vanishing harmonic moments.

The main results of this paper are as follows.

Proposition 3. For any non-degenerate set $S=\left\{z_{0}, z_{1}, \ldots, z_{n}\right\}, n \geq 2$ of points in $\mathbb{C}$,

$$
\operatorname{dim}_{\mathbb{C}} \mathfrak{M}_{n u l l}^{\mathbb{C}}=\left(\begin{array}{c}
n-1 \\
2
\end{array}\right)
$$

Example 2. For $n=3$ the space $\mathfrak{M}_{\text {null }}^{\mathbb{C}}(S)$ is spanned by the complex-valued measure $\tilde{\mu}$ whose densities with respect to the basis of triangles $\Delta_{012}, \Delta_{013}, \Delta_{023}$ are given by:

$$
\left\{\begin{array}{l}
d_{012}=\left(z_{1}-z_{2}\right) /|[012]| \\
d_{013}=\left(z_{3}-z_{1}\right) /|[013]| \\
d_{023}=\left(z_{2}-z_{3}\right) /|[023]|
\end{array},\right.
$$

where $[i, j, k]=\operatorname{det}\left(\begin{array}{ccc}1 & 1 & 1 \\ x_{i} & x_{j} & x_{k} \\ y_{i} & y_{j} & y_{k}\end{array}\right)$ stands for twice the signed area of the triangle with nodes $z_{i}, z_{j}, z_{k}$ and $z_{j}=x_{j}+y_{j} I, I$ being the imaginary unit.

Remark 1. For $S$ non-degenerate, the space $\mathfrak{M}_{\text {null }}^{\mathbb{C}}(S)$ projects isomorphically on the linear subspace of $\mathfrak{M}^{\mathbb{C}}(S)$ spanned by all triangles $\Delta_{0, i, j}$ where $2 \leq i<j \leq n$. In other words, assigning arbitrarily complex-valued densities $d_{0, i, j}, 2 \leq i<j \leq n$ we can uniquely determine the densities $d_{0,1, j}, j=2, \ldots, n$ to get a measure belonging to $\mathfrak{M}_{\text {null }}^{\mathbb{C}}(S)$.

Theorem 1. For any non-degenerate set $S=\left\{z_{0}, z_{1}, \ldots, z_{n}\right\}, n \geq 2$ of points in $\mathbb{C}$

$$
\operatorname{dim}_{\mathbb{R}} \mathfrak{M}_{\text {null }}^{\mathbb{R}}(S)=\left(\begin{array}{c}
n-2 \\
2
\end{array}\right)
$$


Remark 2. For $S$ non-degenerate, the space $\mathfrak{M}_{\text {null }}^{\mathbb{R}}(S)$ projects isomorphically on the linear subspace of $\mathfrak{M}^{\mathbb{R}}(S)$ spanned by all triangles $\Delta_{0, i, j}$ where $3 \leq i<j \leq n$. In other words, arbitrarily real-valued densities $d_{0, i, j}, 3 \leq i<j \leq n$, uniquely determine the densities $d_{0,1, j}, j=2, \ldots, n$ and $d_{0,2, j}, j=3, \ldots, n$ of a measure belonging to $\mathfrak{M}_{\text {null }}^{\mathbb{R}}(S)$.

Theorem 2. For any non-degenerate 5-tuple $S=\left\{z_{0}, z_{1}, z_{2}, z_{3}, z_{4}\right\}$, the space $\mathfrak{M}_{\text {null }}^{\mathbb{R}}(S)$ is spanned by the real measure $\tilde{\mu}$ with densities with respect to the basis of triangles $\Delta_{012}, \Delta_{013}, \Delta_{014}, \Delta_{023}, \Delta_{024}, \Delta_{034}$ given by:

$$
\left\{\begin{array}{l}
d_{012}=\left\|z_{1}-z_{2}\right\|^{2}[134][234] /|[012]| \\
d_{013}=\left\|z_{1}-z_{3}\right\|^{2}[124][234] /|[013]| \\
d_{014}=\left\|z_{1}-z_{4}\right\|^{2}[123][234] /|[014]| \\
d_{023}=-\left\|z_{2}-z_{3}\right\|^{2}[124][134] /|[023]| \\
d_{024}=-\left\|z_{2}-z_{4}\right\|^{2}[134][123] /|[024]| \\
d_{034}=-\left\|z_{3}-z_{4}\right\|^{2}[123][124] /|[034]|
\end{array}\right.
$$

Example 3. For the 5-tuple $\{0,2,3+I, 1+3 I, 2 I\}$ the measure $3 \tilde{\mu}$ is shown in Fig. 2 below. (In this case $3 \tilde{\mu}$ has integer densities which are easier to show TEXnically.)

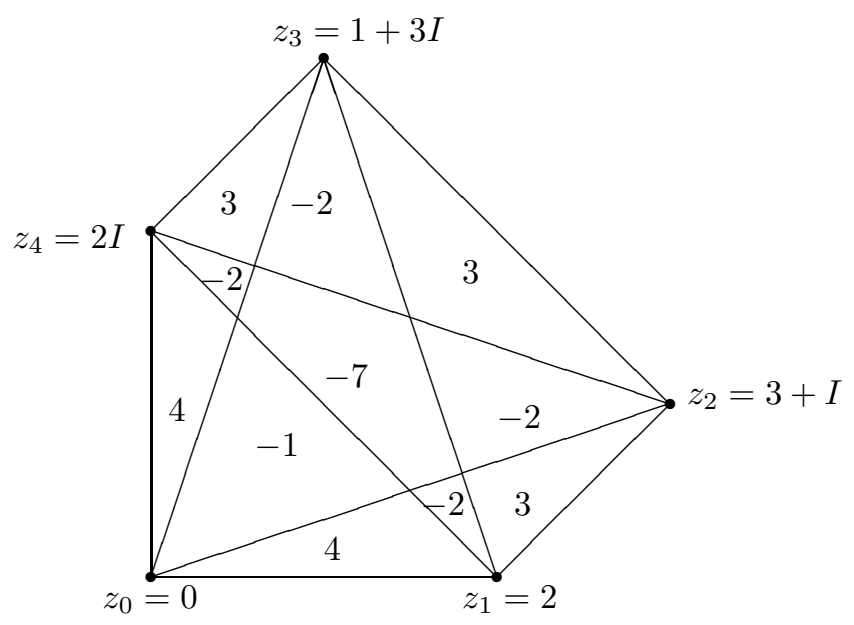

Figure 2. Measure $3 \tilde{\mu}$ spanning $\mathfrak{M}_{\text {null }}^{\mathbb{R}}(0,2,3+I, 1+3 I, 2 I)$.

Remark 3. Suppose that the densities of a polygonal measure $\mu \in \mathfrak{M}_{\text {null }}^{\mathbb{R}}(S)$ with respect to the basic triangles containing a fixed node (say $z_{0}$ ) are known. It is still desirable to find the densities in all its chambers, for instance in view of the classical Problem 1. Here by a chamber we mean a connected component of $\operatorname{conv}(S) \backslash \operatorname{Arr}(S)$, $\operatorname{Arr}(S)$ being the union of all lines connecting pairs of points in $S$. (Integers in Fig. 2 show the densities in the chambers they are placed in.) Each chamber is contained in a number of basic triangles and the density of a given chamber equals the sum of the densities of all basic triangles containing it. Containment of chambers in triangles (and more generally in simplices in $\mathbb{R}^{d}$ ) can be coded by an appropriate incidence matrix whose rows correspond to simplices and columns correspond to chambers. If a simplex contains a chamber then the corresponding entry of the incidence matrix equals 1 , otherwise the entry equals 0 . Examples of incidence matrices are given in the proof of Theorem 3 below. 
This incidence matrix of chambers and simplices in $\mathbb{R}^{d}$ was for the first time studied in [3] and later in [1, 2]. It has rather delicate properties and already the number of chambers is a complicated function of the initial non-degenerate set $S$. In particular, this number can change if we deform $S$ within the class of non-degenerate sets. This observation partially explains why results of the present paper do not automatically solve Problem 1.

Remark 4. Notice that if $S=\left\{z_{0}, \ldots, z_{n}\right\}$ consists of complex numbers having only rational real and imaginary parts then one can choose a basis of $\mathfrak{M}_{\text {null }}^{\mathbb{R}}(S)$ consisting of polygonal measures with integer densities.

Using Example 1 together with Theorem 2 we can prove the following result related to the classical Problem 1 .

Theorem 3. For each $n \geq 6$ there exists $S$, with $|S|=n$, admitting a pair of equipotential polygons. No such $S$ exists if $|S| \leq 5$.

The essential part of the proof of Theorem 3 is to deal with the case $|S|=5$.

Our final result concerns a natural cone spanned by the standard measures of triangles with nodes in $S$. Namely, for an arbitrary non-degenerate set $S=$ $\left\{z_{0}, z_{1}, \ldots, z_{n}\right\}$ denote by $\mathfrak{K}(S) \subset \mathfrak{M}^{\mathbb{R}}(S)$ the $\left(\begin{array}{l}n \\ 2\end{array}\right)$-dimensional cone obtained by taking non-negative linear combinations of the standard measures of all triangles with nodes in $S$. (Recall that $\mathfrak{M}^{\mathbb{R}}(S)$ is the linear span of these measures.)

Theorem 4. Extreme rays of $\mathfrak{K}(S)$ are spanned by (the standard measures) of triangles which do not contain any point of $S$ different from its own nodes. In particular, if $S$ is a convex configuration, (i.e. each $z_{j}$ belongs to the convex hull of $S)$ then every triangle with nodes in $S$ spans an extreme ray of $\mathfrak{K}(S)$.

We finish the introduction with a conjectural description of all faces of $\mathfrak{K}(S)$. We say that a pair of triangles with vertices in $S$ forms a flip if they have a common side and their convex hull is a 4-gon. With any pair of triangles forming a flip we associate their flipped pair obtained by removing the opposite diagonal from their convex hull, see Case a) Fig. 3 below. (On this figure the pairs of triangles $\left(\Delta_{013}, \Delta_{123}\right)$ and $\left(\Delta_{012}, \Delta_{023}\right)$ form a flip and each pair is the flipped one to the other pair.)

Conjecture 1. A collection $C o l$ of triangles having no internal vertices spans a face of $\mathfrak{K}(S)$ if and only if for each pair of triangles from Col forming a flip its flipped pair of triangles is also contained in Col.

The necessity of the stated condition is quite obvious and its sufficiency might follow from the results of [3].

Acknowledgements. B.S. is grateful to the Division of Mathematical Sciences of Nanyang Technological University for hospitality in April 2012 when this project was carried out. D.V.P. is supported by Singapore MOE Tier 2 Grant MOE2011T2-1-090 (ARC 19/11). The authors thank Sinai Robins for helpful discussions. The authors are grateful to the anonymous referee for extremely useful comments on the initial version of this paper.

\section{ProOFs}

Proof of Proposition 1. First, we prove (1.3). Indeed,

$$
\int \frac{d \mu(z)}{(1-u z)^{3}}=\sum_{k \geq 0} u^{k} \int\left(\begin{array}{c}
k+2 \\
2
\end{array}\right) z^{k} d \mu(z)=\sum_{k \geq 0} u^{k}\left(\begin{array}{c}
k+2 \\
2
\end{array}\right) m_{k}(\mu)=\Psi_{\mu}(u),
$$


as required. By [7, (1)], for any $f(z)$ analytic in the closure of $\Delta$, we have

$$
\frac{1}{2 \operatorname{Area} \Delta} \int_{\Delta} f^{\prime \prime}(z) d x d y=\sum_{k=1, j \neq i \in\{1,2,3\} \backslash\{k\}}^{k} \frac{f\left(z_{k}\right)}{\left(z_{k}-z_{i}\right)\left(z_{k}-z_{j}\right)} \text {. }
$$

Applying the latter identity and (1.3) to $f(z)=\frac{1}{2 u^{2}} \frac{1}{1-u z}$, we get the claimed formula.

To prove Proposition 2 we need to recall some basic notions. First we present a description of all linear dependences among the standard measures of all triangles with vertices in a non-degenerate set $S$. Namely, any 4-tuple of points (say, $\left.\left\{z_{0}, z_{1}, z_{2}, z_{3}\right\}\right)$ in $S$ has 4 triangles with vertices at these points. To study linear dependences between these 4 triangles, one has to distinguish between two cases. Consider the convex hull of $\left\{z_{0}, z_{1}, z_{2}, z_{3}\right\}$, which is either a quadrangle or a triangle, see Fig. 3. Obviously, in Case a) we have (up to permutation of the vertices) the equality $\mu_{\Delta_{013}}+\mu_{\Delta_{123}}=\mu_{\Delta_{023}}+\mu_{\Delta_{012}}$. Analogously, in Case b) we have (up to permutation of the vertices) the relation $\mu_{\Delta_{012}}=\mu_{\Delta_{013}}+\mu_{\Delta_{123}}+\mu_{\Delta_{023}}$.

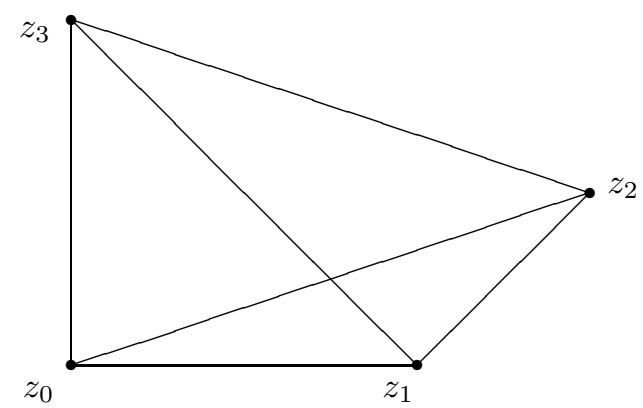

Case a)

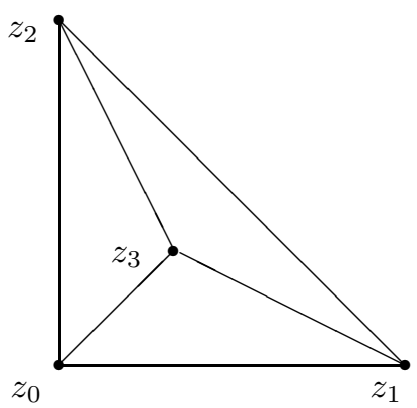

Case b)

FiguRE 3. Linear dependence of 4 triangles spanned by 4 points.

To complete the proof of Proposition 2 we need to show that if $S$ is nondegenerate then the set of (the standard measures of) all triangles containing a given vertex $z_{j} \in S$ spans $\mathfrak{M}^{\mathbb{R}}(S)$ and that this set is linearly independent. The former immediately follows from the discussion preceding Fig. 3, It remains to show the latter. We need more notions.

Definition 3. By a 2-chain $\mathcal{C}^{(2)}$ we mean a formal linear combination

$$
\mathcal{C}^{(2)}=\alpha_{1} \Delta_{1}+\alpha_{2} \Delta_{2}+\ldots+\alpha_{s} \Delta_{s}
$$

of triangles $\Delta_{1}, \ldots \Delta_{s}$ in $\mathbb{C}$ with real or complex coefficients where each triangle is equipped with the standard orientation induced from $\mathbb{C}$.

By using the standard pairing

$$
\left\langle f d x d y, \mathcal{C}^{(2)}\right\rangle=\int_{\mathcal{C}^{(2)}} f d x d y=\sum_{j=1}^{s} \alpha_{j} \int_{\Delta_{j}} f d x d y,
$$

one sees that a 2-chain (2.1) defines a linear functional on the space $\Omega^{(2)}$ of smooth 2 -forms on $\mathbb{C}$.

Definition 4. Analogously, by a 1-chain $\mathcal{C}^{(1)}$ we mean a formal linear combination

$$
\mathcal{C}^{(1)}=\beta_{1} I_{1}+\beta_{2} I_{2}+\ldots+\beta_{t} I_{t}
$$

of oriented finite intervals $I_{1}, \ldots I_{s}$ in $\mathbb{C}$ with real or complex coefficients. 
Again, by using the standard pairing

$$
\left\langle w, \mathcal{C}^{(1)}\right\rangle=\int_{\mathcal{C}^{(1)}} w=\sum_{j=1}^{t} \beta_{j} \int_{I_{j}} w,
$$

where $w$ is an arbitrary smooth 1-form, one sees that a 1-chain (2.2) defines a linear functional on the space $\Omega^{(1)}$ of smooth 1 -forms on $\mathbb{C}$.

Definition 5. For a given triangle $\Delta$ with vertices $a, b, c$ where triple $(a, b, c)$ is counterclockwise oriented we define its boundary $\partial \Delta$ as the sum of three oriented intervals $[a b]+[b c]+[c a]$. As usual, we extend the boundary operator $\partial$ by linearity to the linear space of all 2-chains.

Definition 6. A 2-chain (resp. a 1-chain) is called vanishing if it defines the zero linear functional on $\Omega^{(2)}$ (resp. $\left.\Omega^{(1)}\right)$.

Lemma 1. A 2-chain $\mathcal{C}^{(2)}$ is vanishing if and only if its boundary $\partial \mathcal{C}^{(2)}$ is a vanishing 1-chain.

Proof. Stokes theorem says that $\int_{\partial \Delta} w=\int_{\Delta} d w$, where $w \in \Omega^{(1)}, \Delta$ is an arbitrary triangle, $\partial \Delta$ is its boundary and $d w$ is the differential of $w$. (Recall that if $w=F(x, y) d x+G(x, y) d y$ then $d w=\left(G_{x}^{\prime}-F_{y}^{\prime}\right) d x d y$.) Observe that any 2-form $f(x, y) d x d y$ can be represented as $d w_{x}$ where $w_{x}=F(x, y) d x$ and $F(x, y)$ is the primitive function of $-f(x, y)$ along vertical lines. Analogously, $f(x, y) d x d y$ equals $d w_{y}$ where $w_{y}=G(x, y) d y$ and $G(x, y)$ is the primitive function of $f(x, y)$ along horizontal lines. Thus

$$
\int_{\mathcal{C}^{(2)}} f d x d y=\int_{\partial \mathcal{C}^{(2)}} w_{x}=\int_{\partial \mathcal{C}^{(2)}} w_{y}
$$

If the l.h.s. vanishes for all $f d x d y$ then $\partial \mathcal{C}^{(2)}$ should vanish and vice versa.

Proof of Proposition 2. We need to show that for any non-degenerate $S$ the standard measures of all triangles containing $z_{0}$ are linearly independent. Indeed, by Lemma 1 a 2 -chain $\mathcal{C}^{(2)}$ of triangles vanishes if and only its boundary chain $\partial \mathcal{C}^{(2)}$ vanishes, But if $S$ is non-degenerate then each triangle $\Delta_{0, i, j}$ has its unique edge $\left(z_{i}, z_{j}\right)$ in the boundary and no chain of the form $\beta_{i, j}\left(z_{i}, z_{j}\right)$ with non-trivial $\beta_{i, j}$ can be vanishing. Therefore the standard measures of triangles $\Delta_{0, i, j}$ form a basis in $\mathfrak{M}^{\mathbb{C}}(S)$ and $\mathfrak{M}^{\mathbb{R}}(S)$.

Remark 5. Proposition2 2 has an interesting and immediate corollary, that the linear dependences among the standard measures of all triangles with vertices in $S$ are generated by the linear dependences shown on Fig. 3 which come from all possible 4-tuples of vertices in $S$.

It is a special case of [3, Theorem 1]. (Unfortunately, it seems that a proof of this important statement is missing in the available literature.) J.A. De Loera informed us that it can be derived from results in [8] (e.g. in the plane case one can use Lawson Theorem), or [9].

Proof of Proposition 3. The case $n=2$ is trivial, so we assume $n \geq 3$. Given a non-degenerate $S=\left\{z_{0}, z_{1}, \ldots, z_{n}\right\}$, consider the complex-valued measure $\mu$ obtained by assigning (complex) densities $d_{0 i j}, 1 \leq i<j \leq n$ to triangles $\Delta_{0 i j}$. Set $m_{i, j}=d_{0 i j}$ Area $\Delta_{0 i j}$. Then the normalized generating function $\Psi_{\mu}(u)$ for harmonic moments of $\mu$ is given by

$$
\begin{array}{r}
\Psi_{\mu}(u)=\sum_{1 \leq i<j \leq n} d_{0 i j} \Psi_{\Delta_{0 i j}}(u)=\sum_{1 \leq i<j \leq n} \frac{m_{i j}}{\left(1-z_{0} u\right)\left(1-z_{i} u\right)\left(1-z_{j} u\right)} \\
=\frac{1}{1-z_{0} u} \frac{P(u)}{\prod_{i=1}^{n}\left(1-z_{i} u\right)}
\end{array}
$$


where $P(u)$ is a polynomial of degree at most $n-2$. Its coefficients at $1, u, u^{2}, \ldots$, $u^{n-2}$ are the consecutive entries of the vector $\mathcal{M}_{n}^{\mathbb{C}} \cdot \mathbf{m}_{n}$, where

$$
\mathbf{m}_{n}=\left(m_{12}, m_{13}, \ldots m_{n-1, n}\right)^{\top}
$$

with $m_{i, j}$ 's ordered lexicographically, and $\mathcal{M}_{n}^{\mathbb{C}}$ is the $(n-1) \times\left(\begin{array}{l}n \\ 2\end{array}\right)$-matrix with columns corresponding to $m_{i, j}$. Such a column contains consecutive elementary symmetric functions of the $(n-2)$-tuple $\left(-z_{1},-z_{2}, \ldots-\hat{z}_{i}, \ldots,-\hat{z}_{j}, \ldots,-z_{n}\right)$, where $\hat{z}_{i}$ and $\hat{z}_{j}$ stands for the omission of these points.

Example 4. For $n=4$ the coefficients at $\left(1, u, u^{2}\right)$ of the numerator $P(u)$ of (2.3) are the consecutive entries of the vector $\mathcal{M}_{4}^{\mathbb{C}} \cdot \mathbf{m}_{4}$ where

$$
\begin{aligned}
\mathbf{m}_{4} & =\left(m_{12}, m_{13}, m_{14}, m_{23}, m_{24}, m_{34}\right)^{\top} \\
\mathcal{M}_{4}^{\mathbb{C}} & =\left(\begin{array}{cccccc}
1 & 1 & 1 & 1 & 1 & 1 \\
-z_{3}-z_{4} & -z_{2}-z_{4} & -z_{2}-z_{3} & -z_{1}-z_{4} & -z_{1}-z_{3} & -z_{1}-z_{2} \\
z_{3} z_{4} & z_{2} z_{4} & z_{2} z_{3} & z_{1} z_{4} & z_{1} z_{3} & z_{1} z_{2}
\end{array}\right) .
\end{aligned}
$$

In other words,

$$
P(u)=\sum_{\substack{1 \leq i<j \leq 4 \\ k<\ell,\{i j\} \cap\{k \ell\}=\emptyset}}\left(m_{i j}-\left(z_{i}+z_{j}\right) m_{k \ell} u+z_{i} z_{j} m_{k \ell} u^{2}\right) .
$$

Consider the maximal minor $\operatorname{Min}_{n}^{\mathbb{C}}$ of $\mathcal{M}_{n}^{\mathbb{C}}$ formed by the columns corresponding to $m_{12}, \ldots, m_{1, n}$, i.e. the first $n-1$ columns of $\mathcal{M}_{n}^{\mathbb{C}}$.

Lemma 2. $\operatorname{det}_{n}=(-1)^{n-1} \operatorname{det}\left(M i n_{n}^{\mathbb{C}}\right)=(-1)^{n-1} \prod_{2 \leq i<j \leq n}\left(z_{i}-z_{j}\right)$.

Proof. Indeed, the degree of $\operatorname{det}\left(\operatorname{Min}_{n}^{\mathbb{C}}\right)$ as a polynomial in $z_{2}, \ldots, z_{n}$ equals $\left(\begin{array}{c}n-1 \\ 2\end{array}\right)$. We need to show that it vanishes if and only if $z_{i}=z_{j}$. The 'if' part is obvious since the column corresponding to $m_{1, i}$ will coincide with the column corresponding to $m_{1, j}$. To see the remaining part, argue by contradiction and assume that $\left(\alpha_{12}, \ldots, \alpha_{1 n}\right)$ is a nontrivial linear dependence among the columns of $\operatorname{Min}_{n}^{\mathbb{C}}$. The $1 k$-th column consists of the coefficients of the polynomial $g_{1 k}(u)=\frac{\prod_{j=1}^{n}\left(1-z_{j} u\right)}{1-z_{k} u}$, and our linear dependence is a linear dependence among such polynomials. Evaluate these at $\frac{1}{z_{j}}$ and note that $g_{1 k}\left(\frac{1}{z_{j}}\right)$ vanish whenever $k \neq j$. Thus $\alpha_{1 j}=0$, a contradiction. Thus $\operatorname{det}\left(\operatorname{Min}_{n}^{\mathbb{C}}\right)$ is divisible by $\prod_{2<i<j<n}\left(z_{i}-z_{j}\right)$. Substituting $z_{2}=0, z_{3}=1, \ldots, z_{n}=n-2$ we can check that the normalizing factor equals $(-1)^{n-1}$.

Remark 6. By using Cramer's rule, it is not difficult to give an explicit formula for the inverse $\left(\operatorname{Min}_{n}^{\mathbb{C}}\right)^{-1}$.

From Lemma 2 we know that for any, not necessarily non-degenerate, $S=$ $\left\{z_{0}, z_{1}, \ldots, z_{n}\right\}$ with pairwise distinct points the rank of $\mathcal{M}_{n}^{\mathbb{C}}$ equals $n-1$. Thus the kernel of $\mathcal{M}_{n}^{\mathbb{C}}$, which by definition coincides with $\mathfrak{M}_{\text {null }}^{\mathbb{C}}(S)$, has dimension $\left(\begin{array}{l}n \\ 2\end{array}\right)-(n-1)=\left(\begin{array}{c}n-1 \\ 2\end{array}\right)$.

Proof of Theorem 1 1 . The space $\mathfrak{M}_{\text {null }}^{\mathbb{R}}(S) \subset \mathfrak{M}_{\text {null }}^{\mathbb{C}}(S)$ is the maximal by inclusion real subspace of the complex kernel. In other words, it can be interpreted as the real kernel of the real matrix $\mathcal{M}_{n}^{\mathbb{R}}$ obtained by taking the real and imaginary parts of all rows of $\mathcal{M}_{n}^{\mathbb{C}}$.

The case $n=2$ is trivial. The case $n=3$ can be dealt with by explicitly computing the kernel of $\mathfrak{M}_{3}^{\mathbb{C}}$ and seeing that it does not contain real vectors if $S$ is non-degenerate. Thus we assume $n \geq 4$. Since the first row of $\mathcal{M}_{n}^{\mathbb{C}}$ equals $(1,1, \ldots, 1)$ the matrix $\mathcal{M}_{n}^{\mathbb{R}}$ has size $(2 n-3)\left(\begin{array}{c}n \\ 2\end{array}\right)$, see (2.4). Ordering $m_{i j}$ 's lexicographically, consider the maximal minor $\operatorname{Min}_{n}^{\mathbb{R}}$ of $\mathcal{M}_{n}^{\mathbb{R}}$ formed by the columns 
corresponding to $(2 n-3)$ variables $m_{12}, m_{13}, \ldots, m_{1 n}, m_{23}, m_{24}, \ldots, m_{2 n}$, i.e. the first $(2 n-3)$ columns of $\mathcal{M}_{n}^{\mathbb{R}}$.

Lemma 3. $\operatorname{det} \operatorname{Min}_{n}^{\mathbb{R}}=C[123][124] \cdots[12 n] \prod_{3 \leq i<j \leq n}\left|z_{i}-z_{j}\right|^{2}, \quad 0 \neq C \in \mathbb{R}$.

Proof. We begin by showing that $\Theta:=\operatorname{det} M i n_{n}^{\mathbb{R}}$ is divisible by $[12 k]$ for any $3 \leq$ $k \leq n$. As $[12 k]$ is an irreducible quadratic polynomial in $x_{1}, x_{2}, x_{k}$ and $y_{1}, y_{2}, y_{k}$, it suffices to show that vanishing of $[12 k]$ implies vanishing of $\Theta$. Vanishing of $[12 k]$ is equivalent to existence of $a \in \mathbb{R}$ satisfying $z_{k}=a z_{1}+(1-a) z_{2}$. The latter implies that $\operatorname{Min}_{n}^{\mathbb{R}}$ has linearly dependent columns $12,1 k$, and $2 k$. Indeed, they consist, respectively, of the coefficients of

$$
\begin{aligned}
& g_{12}(u)=\left(1-a z_{1} u-(1-a) z_{2} u\right) \times\left(1-z_{3} u\right) \ldots\left(1-z_{k-1} u\right)\left(1-z_{k+1} u\right) \ldots\left(1-z_{n} u\right) \\
& g_{1 k}(u)=\left(1-z_{2} u\right) \times \quad\left(1-z_{3} u\right) \ldots\left(1-z_{k-1} u\right)\left(1-z_{k+1} u\right) \ldots\left(1-z_{n} u\right) \\
& g_{2 k}(u)=\left(1-z_{1} u\right) \times \quad\left(1-z_{3} u\right) \ldots\left(1-z_{k-1} u\right)\left(1-z_{k+1} u\right) \ldots\left(1-z_{n} u\right)
\end{aligned}
$$

which are linearly dependent: $g_{12}(u)=(a-1) g_{1 k}(u)-a g_{2 k}(u)$.

To show that $\Theta$ is divisible by $\left|z_{i}-z_{j}\right|^{2}=\left(z_{i}-z_{j}\right)\left(\bar{z}_{i}-\bar{z}_{j}\right)$ for any $3 \leq i<j \leq n$, observe that $z_{i}=z_{j}$ implies $g_{k i}(u)=g_{k j}(u)$ for $k=1,2$.

It remains to see that $\Theta$ is not identically 0 . Arguing by contradiction, let $\left(\alpha_{12}, \alpha_{13}, \ldots, \alpha_{1 n}, \alpha_{23}, \ldots, \alpha_{2 n}\right)$ be the coefficients of a nontrivial real linear dependence among the columns of $\operatorname{Min}_{n}^{\mathbb{R}}$. The latter columns correspond to the coefficients of $g_{i j}(u)$. Evaluating these at $u=\frac{1}{z_{k}}$, for $3 \leq k \leq n$, makes all of them but $g_{1 k}$ and $g_{2 k}$ vanish. Thus

$\alpha_{1 k} g_{1 k}\left(z_{k}^{-1}\right)+\alpha_{2 k} g_{2 k}\left(z_{k}^{-1}\right)=0, \quad$ implying $\alpha_{1 k}=-\alpha_{2 k} \frac{z_{k}-z_{2}}{z_{k}-z_{1}} \quad$ and $\frac{z_{k}-z_{2}}{z_{k}-z_{1}} \in \mathbb{R}$.

A direct computation shows that the rightmost relation is equivalent to $[12 k]=0$, a contradiction.

Lemma 3 implies that for any non-degenerate $S$ the matrix $\mathcal{M}_{n}^{\mathbb{R}}$ has rank equal to $2 n-3$. Therefore, $\operatorname{dim} \mathfrak{M}_{\text {null }}^{\mathbb{R}}(S)=\left(\begin{array}{c}n \\ 2\end{array}\right)-(2 n-3)=\left(\begin{array}{c}n-2 \\ 2\end{array}\right)$.

Proof of Theorem 2. For $S=\left\{0, z_{1}, z_{2}, z_{3}, z_{4}\right\}$ the space $\mathfrak{M}_{n u l l}^{\mathbb{R}}(S)$ is given by the system

$$
\begin{gathered}
\mathcal{M}_{4}^{\mathbb{R}} \cdot \mathbf{m}_{4}=0, \\
\mathcal{M}_{4}^{\mathbb{R}}=\left(\begin{array}{cccccc}
1 & \multicolumn{4}{c}{\text { where } \mathbf{m}_{4}=\left(m_{12}, m_{13}, m_{14}, m_{23}, m_{24}, m_{34}\right)^{\top} \text { and }} \\
-x_{3}-x_{4} & -x_{2}-x_{4} & -x_{2}-x_{3} & -x_{1}-x_{4} & -x_{1}-x_{3} & -x_{1}-x_{2} \\
-y_{3}-y_{4} & -y_{2}-y_{4} & -y_{2}-y_{3} & -y_{1}-y_{4} & -y_{1}-y_{3} & -y_{1}-y_{2} \\
x_{3} x_{4}-y_{3} y_{4} & x_{2} x_{4}-y_{2} y_{4} & x_{2} x_{3}-y_{2} y_{3} & x_{1} x_{4}-y_{1} y_{4} & x_{1} x_{3}-y_{1} y_{3} & x_{1} x_{2}-y_{1} y_{2} \\
x_{3} y_{4}+x_{4} y_{3} & x_{2} y_{4}+x_{4} y_{2} & x_{2} y_{3}+x_{3} y_{2} & x_{1} y_{4}+x_{4} y_{1} & x_{1} y_{3}+x_{3} y_{1} & x_{1} y_{2}+x_{2} y_{1}
\end{array}\right) .
\end{gathered}
$$

Recall that a $k \times(k+1)$-matrix $T$ of rank $k$ has right kernel spanned by the vector $\left(T^{(1)}, \ldots, T^{(k+1)}\right)$, where $T^{(j)}$ is the minor of $T$ with $j$ th column removed multiplied by $(-1)^{j}$. Thus (2.4) has a unique (up to a scaling) solution of the form:

$$
\left\{\begin{array}{ll}
m_{12}= & \left\|z_{1}-z_{2}\right\|^{2}[134][234] \\
m_{13}= & \left\|z_{1}-z_{3}\right\|^{2}[124][234] \\
m_{14}= & \left\|z_{1}-z_{4}\right\|^{2}[123][234] \\
m_{23}=-\left\|z_{2}-z_{3}\right\|^{2}[124][134] \\
m_{24}=-\left\|z_{2}-z_{4}\right\|^{2}[134][123] \\
m_{34}=-\left\|z_{3}-z_{4}\right\|^{2}[123][124]
\end{array} .\right.
$$

It is easy to prove this. We give a sketch here for $m_{12}$. Note that $m_{12}$ equals to the determinant of the matrix $A^{(12)}$ obtained from $\mathcal{M}_{4}^{\mathbb{R}}$ by removing the 1 st 
column. Then, $\operatorname{det} A^{(12)}$ is divisible by $\left\|z_{1}-z_{2}\right\|^{2}$, as the rank of $A^{(12)}$ drops when $z_{1}=z_{2}$, and as $\left\|z_{1}-z_{2}\right\|^{2}=\left(z_{1}-z_{2}\right)\left(\bar{z}_{1}-\bar{z}_{2}\right)$ is the product of two irreducible polynomials with complex coefficients.

Similarly, $\operatorname{det} A^{(12)}$ is divisible by [234] (and a very similar argument applies to [134]). To see this, note that, as [234] is irreducible, it suffices to show that its vanishing implies vanishing of $\operatorname{det} A^{(12)}$. To this end, assume that $z_{4}=a z_{2}+(1-$ a) $z_{3}$, with $a \in \mathcal{R}$, and make this substitution into $A^{(12)}$. The last 3 columns of $A^{(12)}$ become

$$
\left(\begin{array}{ccc}
1 & 1 & 1 \\
-a x_{2}+a x_{3}-x_{1}-x_{3} & -x_{1}-x_{3} & -x_{1}-x_{2} \\
-a y_{2}+a y_{3}-y_{1}-y_{3} & -y_{1}-y_{3} & -y_{1}-y_{2} \\
a x_{1} x_{2}-a x_{1} x_{3}-a y_{1} y_{2}+a y_{1} y_{3}+x_{1} x_{3}-y_{1} y_{3} & x_{1} x_{3}-y_{1} y_{3} & x_{1} x_{2}-y_{1} y_{2} \\
a x_{1} y_{2}-a x_{1} y_{3}+a x_{2} y_{1}-a x_{3} y_{1}+x_{1} y_{3}+x_{3} y_{1} & x_{1} y_{3}+x_{3} y_{1} & x_{1} y_{2}+x_{2} y_{1}
\end{array}\right) .
$$

They are linearly dependent with coefficients $(1, a-1,-a)$.

Proof of Theorem 3. To prove the first part, we recall that Example 1 settles the case $|S|=6$. To settle the case $|S|=6+q$, we modify the latter Example. Add $q$ points $P_{1}, \ldots, P_{q}$ outside $\operatorname{conv}(T)$, so that so that $P_{1}, \ldots, P_{q}$ and $\sqrt{3} \pm I$ are in the convex position, and $Q$ is the resulting convex $q+2$. Then $F \cup Q$ and $F^{\prime} \cup Q$ are equipotential $(6+q)$-gons, by additivity of the measure.

To prove the second part, we have consider the cases $|S|=3,4,5$, one by one. Cases $|S|=3,4$ follow from Theorem 1

It remains to deal with the only non-trivial case $|S|=5$. We have to consider the incidence matrices between the chambers and the basic simplices for all possible non-degenerate 5 -tuples of points $S$. One can easily see that for non-degenerate 5 tuples there are (up to permutation of the vertices) only 3 different cases to consider depending on the shape of $\operatorname{conv}(S)$ which can be a 5-gon, a 4-gon, or a triangle. The corresponding incidence matrices $I n c_{1}, I n c_{2}, I n c_{3}$ are given below using the labeling presented in Fig. 4 and 5 for these cases. (Greek letters in Fig. 4 denote the vertices of the inner 5-gon. They will be needed below.) We show that in none of these case one can find a pair of equipotential polygons.

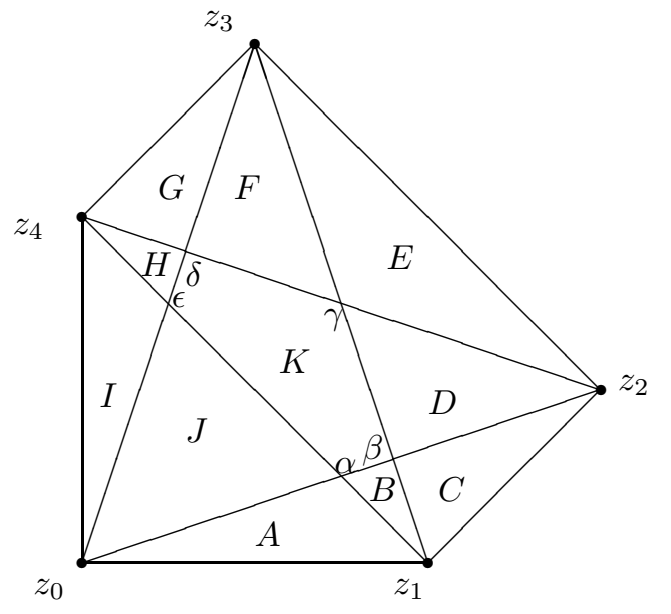

Figure 4. Chambers and their labeling for $\operatorname{conv}(S)$ a 5 -gon. 


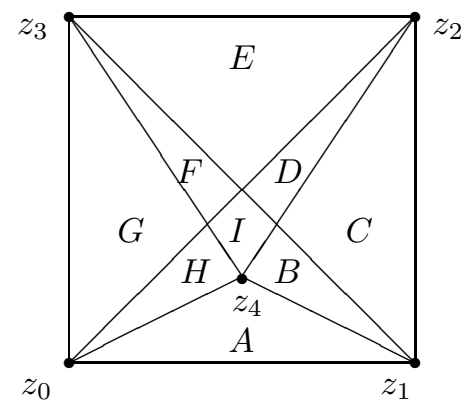

4-gon

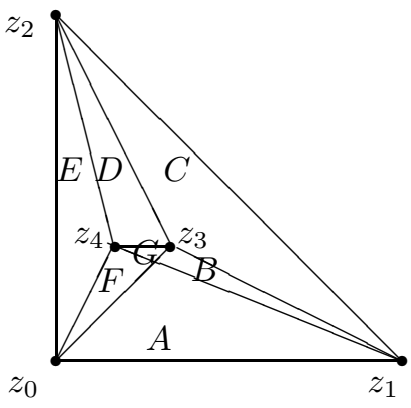

triangle

Figure 5. Chambers and their labeling for $\operatorname{conv}(S)$ a 4-gon or a triangle.

\begin{tabular}{|c|c|c|c|c|c|c|c|c|c|c|c|}
\hline & $A$ & B & $C$ & $D$ & $E$ & $F$ & $G$ & $H$ & $I$ & $J$ & $K$ \\
\hline$\Delta_{012}$ & 1 & 1 & 1 & 0 & 0 & 0 & 0 & 0 & 0 & 0 & 0 \\
\hline$\Delta_{013}$ & 1 & 1 & 0 & 0 & 0 & 1 & 0 & 0 & 0 & 1 & 1 \\
\hline$={ }^{\Delta_{014}}$ & 1 & 0 & 0 & 0 & 0 & 0 & 0 & 0 & 1 & 1 & 0 \\
\hline$\Delta_{023}$ & 0 & 0 & 0 & 1 & 1 & 1 & 0 & 0 & 0 & 1 & 1 \\
\hline$\Delta_{024}$ & 0 & 0 & 0 & 1 & 0 & 0 & 0 & 1 & 1 & 1 & 1 \\
\hline$\Delta_{034}$ & 0 & 0 & 0 & 0 & 0 & 0 & 1 & 1 & 1 & 0 & 0 \\
\hline
\end{tabular}

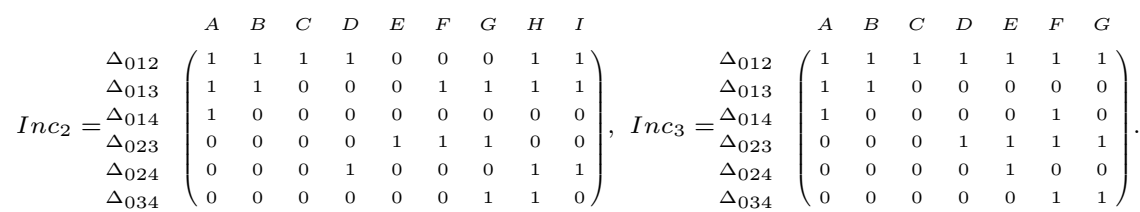

For brevity, we introduce notation $\frac{1}{2}|[K]|$ for the area of a polygon $K$. First, we need an elementary

Lemma 4. For an arbitrary triangle $\Delta_{\alpha \beta \gamma}$ and arbitrary secants $\alpha \epsilon, \beta \delta$, see Fig. 6 the area of triangle $\Delta_{\alpha \beta \zeta}$ is bigger than that of $\Delta_{\epsilon \delta \zeta}$ :

$$
\left|\left[\Delta_{\alpha \beta \zeta}\right]\right|>\left|\left[\Delta_{\epsilon \delta \zeta}\right]\right| \text {. }
$$

Proof. Indeed, draw the line $\alpha \kappa$ parallel to $\beta \gamma$ and extend $\beta \delta$ till it hits $\alpha \kappa$. (The intersection point of the latter lines is denoted by $\eta$.) Triangles $\Delta_{\alpha \beta \zeta}$ and $\Delta_{\eta \epsilon \zeta}$ have equal area. Indeed, they are obtained from $\Delta_{\alpha \beta \eta}$ and $\Delta_{\alpha \epsilon \eta}$, respectively, by removing $\Delta_{\alpha \zeta \eta}$. Notice that $\Delta_{\alpha \beta \eta}$ and $\Delta_{\alpha \epsilon \eta}$ have the same base $\alpha \eta$ and equal heights.

Case 2. Using labeling on the left part of Fig. 5]and (1.4) we conclude that densities $d_{012}, d_{014}, d_{023}, d_{034}$ are positive while $d_{013}, d_{024}$ are negative. From chambers $E$ and $C$ we conclude $d_{023}=d_{012}=1$. Then from chamber $D$ we have that either $d_{024}=-1$ or -2 . The second case leads to $d_{013}=0$, contradiction. Thus $d_{024}=-1$ which from chamber $I$ gives $d_{013}=-1$. Finally, $d_{034}=d_{014}=1$. Thus chambers $A, C, E, G$ have density 1 , chamber $I$ has density -1 and the remaining chambers have vanishing density. We need to show that $|[I]|<|[A]|+|[C]|+|[E]|+|[G]|$. We will show that actually $|[I]|<|[C]|+|[G]|$. Cut $I$ into two triangles by drawing its diagonal connecting $z_{4}$ with non-neighboring vertex $p$ of $I$ (lying strictly above $z_{4}$ in the left part of Fig. (5). Extending $z_{3} p$ and $z_{0} z_{4}$ we get a triangle containing $G$ and the left half of $I$ and we can apply Lemma 4. Analogously, extending $z_{2} p$ 
$\kappa$

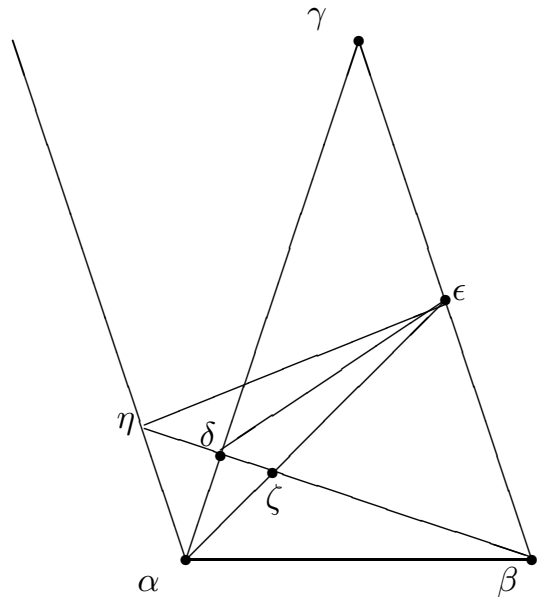

FiguRE 6. Illustration to Lemma 4

and $z_{1} z_{4}$ we get a triangle containing $C$ and the right half of $I$ and we can apply Lemma 4. Thus the required measure does not exist.

Case 3. Using labeling on the right part of Fig. 5 and formulas (1.4) we again conclude that densities $d_{012}, d_{014}, d_{024}$ are negative, while $d_{013}, d_{023}, d_{034}$ are positive. Similar considerations as above give $d_{012}=d_{014}=d_{024}=-1$ and $d_{013}=d_{023}=$ $d_{034}=1$. Thus, the densities of $A, C, E$ are -1 and the density of $G$ is 1 . In fact, $|[G]|<|[A]|$ already. Indeed, extending the interval $z_{0} z_{4}$ and $z_{1} z_{3}$ till they intersect at a point, say $p$ we get the triangle $z_{0} z_{1} p$ to which we apply Lemma 4 . Thus the required measure does not exist.

Case 1. Using labeling on Fig. 4 and (1.4) we see that densities $d_{012}, d_{014}$, $d_{023}, d_{034}$ are positive while $d_{013}, d_{024}$ are negative. Assuming that the densities of all chambers attain only values $0, \pm 1$ and looking at chambers $C, E, G$ we get that $d_{023}=d_{012}=d_{034}=1$. Looking at chamber $D$ we conclude that $d_{024}=-1$. (It might be equal -2 as well but then looking at chamber $K$ we have to conclude that $d_{013}=0$ which is impossible.) From chamber $K$ we get $d_{013}=-1$ and from chamber $I$ we get $d_{014}=1$. Thus the density in chambers $A, C, E, G, I$ equals 1 , in chamber $K$ it equals -1 and it vanishes in the remaining chambers. Notice that the total mass of the measure should vanish. To see that this cannot happen, we show that $|[K]|<|[A]|+|[C]|+|[E]|+|[G]|+|[I]|$. Using Lemma 4 we conclude that $|[A]|>\left|\left[\Delta_{\alpha \beta \epsilon}\right]\right|,|[C]|>\left|\left[\Delta_{\alpha \beta \gamma}\right]\right|,|[E]|>\left|\left[\Delta_{\beta \gamma \delta}\right]\right|,|[G]|>\left|\left[\Delta_{\delta \epsilon \gamma}\right]\right|$, and $|[I]|>\left|\left[\Delta_{\epsilon \alpha \delta}\right]\right|$, see Fig. 4. Triangles $\Delta_{\alpha \beta \epsilon}, \Delta_{\alpha \beta \gamma}, \Delta_{\beta \gamma \delta}, \Delta_{\delta \epsilon \gamma}, \Delta_{\epsilon \alpha \delta}$ pairwise overlap. These overlapping consists of 5 smaller triangles inside $K$. The complement in $K$ to the union of triangles $\Delta_{\alpha \beta \epsilon}, \Delta_{\alpha \beta \gamma}, \Delta_{\beta \gamma \delta}, \Delta_{\delta \epsilon \gamma}, \Delta_{\epsilon \alpha \delta}$ is a small 5-gon inside $K$. Now we can use these 5 small triangles to cover the small 5 -gon inside $K$. We get exactly the same situation as the original one and we can apply the same argument as we did and cover a substantial part of the small 5-gon etc. Continuing this process we will in infinitely many steps exhaust the original 5 -gon $K$. Thus the required measure does not exist.

To prove Theorem 4 we need the following observation.

Lemma 5. The convex hull of the standard measures of 4 triangles as in Case a) Fig. 3, i.e. two pairs forming a flip is a plane quadrangle. The convex hull of the standard measures of 4 triangles as in Case b) Fig. 3 is a plane triangle.

Proof. Obvious from the relations given above Fig. 3 . 
Proof of Theorem 4. Indeed if a triangle $\Delta$ contains an interior point other than its vertices than $\mu_{\Delta}$ is the sum of three triangles in which it is subdivided by an inner vertex, see Lemma 5. (Recall that $S$ is non-degenerate by assumption.) Thus $\mu_{\Delta}$ is not an extreme ray. On the other hand, assume that no point in $S$ other than its vertices is contained in $\Delta$ and $\mu_{\Delta}$ is a linear combination of the standard measures of some other triangles with vertices in $S$ with positive coefficients. Since no such triangle can be contained strictly inside $\Delta$ by assumption and all coefficients are positive we get that any such linear combination necessarily has positive density somewhere outside $\Delta$, contradiction.

\section{OPEN PROBLEMS}

1. Theorem 1 gives the dimension of $\mathfrak{M}_{n u l l}^{\mathbb{R}}(S)$ for non-degenerate $S$. Its dimension for arbitrary $S$ is unclear. On one hand, if $S$ is degenerate then $\operatorname{dim} \mathfrak{M}^{\mathbb{R}}(S)$ decreases. On the other hand, the number of equations imposed on the densities might also decrease. It seems highly plausible that $\operatorname{dim} \mathfrak{M}_{n u l l}^{\mathbb{R}}(S)$ for an arbitrary $S$ depends only on non-oriented matroid associated to this set, see e.g. [10]. An algorithm calculating this dimension is given in [2].

2. Besides the cone $\mathfrak{K}(S) \subset \mathfrak{M}^{\mathbb{R}}(S)$ one can introduce a more important, bigger, cone $\mathfrak{K}_{\text {pos }}(S) \subset \mathfrak{M}^{\mathbb{R}}(S)$ where $\mathfrak{K}_{\text {pos }}(S) \supset \mathfrak{K}(S)$ consists of all non-negative measures from $\mathfrak{M}^{\mathbb{R}}(S)$.

Conjecture 2. The combinatorial structure of $\mathfrak{K}_{\text {pos }}(S)$ depends only on the oriented matroid associated to $S$.

Already for generic configurations $S$ with 6 points the combinatorial structure of $\mathfrak{K}_{\text {pos }}(S)$ and, in particular, the set of its extreme rays seems to be quite complicated. We plan to study this fascinating subject in the future.

3. Notice that we have a natural linear map $\Psi_{\mu}: \mathfrak{M}^{\mathbb{R}}(S) \rightarrow$ Rat $_{n}$ obtained by associating to each measure $\mu \in \mathfrak{M}^{\mathbb{R}}(S)$ its normalized generating function (1.2). Here $R_{a t}$ is the linear space of rational functions of the form $R(u)=$ $\frac{P(u)}{\prod_{j=0}^{n}\left(1-z_{j} u\right)}, \quad \operatorname{deg} P(u) \leq n-2$ having real constant term. Obviously, $\operatorname{dim}$ Rat $_{n}=$ $2 n-3$ and using Theorem 1 we obtain that $\mathfrak{M}^{\mathbb{R}}(S)$ is mapped onto Rat $_{n}$. The following question is very natural in connection with the inverse problem for the class of non-negative measures.

Problem 3. Describe the extreme rays/faces of the image cones $\Psi_{\mu}(\mathfrak{K}(S))$ and $\Psi_{\mu}\left(\mathfrak{K}_{\text {pos }}(S)\right.$ in $\operatorname{Rat}_{n}$.

4. We have an example of a pair of equipotential polygons with $|S|=6$, see Fig. 1]

Problem 4. Describe all 6-tuples $S$ admitting a pair of equipotential polygons.

\section{REFERENCES}

[1] T. Alekseyevskaya, Bases in systems of simplices and chambers, arXiv: math/9707218 1.

[2] T. Alekseyevskaya, Combinatorial bases in systems of simplices and chambers, Discrete Mathematics 157 (1996) 15-37.

[3] T. V. Alekseyevskaya, I. M. Gelfand, A. V. Zelevinsky, An arrangement of real hyperplanes and the partition function connected with it, Soviet Math. Doklady 36 (1988) 589-593.

[4] M. Andersson, M. Passare and R. Sigurdsson, Complex Convexity and Analytic Functionals, Progres s in Math. 225 Birkhäuser, 2004, vii +160 .

[5] M. A. Brodsky. On the uniqueness of the inverse potential problem for homogeneous polyhedrons, SIAM Journal on Applied Mathematics, 46, No. 2 (Apr., 1986), 345-350.

[6] M. A. Brodsky and V. N. Strakhov, On the uniqueness of the inverse logarithmic potential problem, SIAM Journal on Applied Mathematics, 46, No. 2 (Apr., 1986), 324-344.

[7] Ph. J. Davis. Triangle formulas in the complex plane. Math. Comp., 18:569-577, 1964. 
[8] J. A. De Loera, S. Hoşten, F. Santos, B. Sturmfels, The polytope of all triangulations of a point configuration, Documenta Mathematica 1 (1996) 103-119

[9] J. A. De Loera, J. Rambau, F. Santos, Triangulations Structures for Algorithms and Applications, Series: Algorithms and Computation in Mathematics, Vol. 25 1st Edition., Springer 2010.

[10] I. M. Gelfand, V. V. Serganova, Combinatorial geometries and toric strata on compact homogeneous manifolds, Soviet Math.Surv, vol. 42(2) 1987, 107-134.

[11] B. Gustafsson, C. He, P. Milanfar, M. Putinar: Reconstructing planar domains from their moments, Inverse Problems 16 (2000), 1053-1070.

[12] G. Golub, B. Gustafsson, P. Milanfar, P. Putinar, J. Varah: Shape reconstruction from moments: theory, algorithms, and applications, SPIE Proceedings vol. 4116 (2000), Advanced Signal Processing, Algorithms, Architecture, and Implementations X (Franklin T. Luk, ed.) $406-416$.

[13] N. Gravin, J. Lassere, D. V. Pasechnik, S. Robins, The inverse moment problem for convex polytopes. Discrete and Comp. Geometry, 48 (2012), 596-621.

[14] N. Gravin, D. V. Pasechnik, B. Shapiro, M. Shapiro, On moments of a polytope, arXiv:1210.3193, submitted.

[15] P. S. Novikov, On the uniqueness of the solution of the inverse potential problem, Doklady AN SSSR, 18 (1938), 165-168. (In Russian.)

School of Physical and Mathematical Sciences, Nanyang Technological UniverSity, 21 Nanyang Link, 637371 Singapore

E-mail address: dima@ntu.edu.sg

Department of Mathematics, Stockholm University, SE-106 91 Stockholm, Sweden E-mail address: shapiro@math.su.se 